\title{
Diet and Neuroimaging Markers of Cerebrovascular Disease
}

\author{
Yian Gu • Nikolaos Scarmeas
}

Published online: 17 April 2013

(C) Springer Science+Business Media New York 2013

\begin{abstract}
Stroke is one of the leading causes of longterm disability and mortality. Mounting evidence from observational studies suggests that among lifestyle factors, diet may play an important role for the prevention of stroke. Neuroimaging markers, particularly white matter hyperintensity (WMH) and brain infarcts (BI), are more sensitive measurements of cerebrovascular disease than clinical assessments. We reviewed published observational and clinical studies that evaluate the association between dietary factors and WMH and BI. The few existing studies examined only a handful individual nutrients or foods (dietary intake of alcohol, B vitamins, fish, choline, serum markers of antioxidants) and a few food groups, Mediterranean-style diet, and nutrient biomarker patterns. Findings from these studies are inconclusive either due to conflicting results from different studies or due to lack of replication. Further studies are necessary to replicate the existing findings. Many other foods or nutrients or dietary patterns may worth investigating and longitudinal studies are needed.
\end{abstract}

Keywords Diet · Nutrition · Nutrients · Foods · Mediterranean diet . White matter hyperintensity . Stroke $\cdot$ Infarct

\footnotetext{
Y. Gu $\cdot$ N. Scarmeas

Department of Neurology, Gertrude H. Sergievsky Center, Taub Institute for Research in Alzheimer's Disease and the Aging Brain, Columbia University, New York, NY, USA

N. Scarmeas

National and Kapodistrian University of Athens Medical School, Athens, Greece

N. Scarmeas $(\triangle)$

Columbia University Medical Center, 622 W 168th St., P\&S \#16, New York, NY 10032, USA

e-mail: ns257@columbia.edu
}

\section{Introduction}

Stroke is one of the leading causes of serious, long-term disability and mortality in the United States [1]. Mounting evidence from observational studies suggests that diet is one of the important lifestyle factors for the prevention of stroke [2]. However, the mechanisms for the relationship between diet and stroke have not been fully explored in the past, partly due to the limited availability of methods that allow a sensitive and accurate measurement of cerebrovascular disease.

Recent advances in magnetic resonance imaging (MRI) techniques have made it possible to visualize and to quantify the structural changes of the brain [3••]. Neuroimaging markers are extremely sensitive measurements of changes occurring in the aging brain. Therefore, using these neuroimaging markers could help to uncover associations between diet and cerebrovascular diseaserelated brain dysfunctions that might not be detectable using rough clinical stroke outcomes. For example, a population-based, longitudinal study demonstrated that $18 \%$ of the elderly study participants developed incident brain infarct on follow-up MRI scan 5 years later, whereas nearly $90 \%$ of those cases had no documented transient ischemic attack or clinical stroke between the scans [4]. Another population-based study reported low specificity $(79 \%)$ and sensitivity (32\%) of self-reported stroke using brain MRI as the standard [5].

Two major neuroimaging markers of cerebrovascular disease, i.e., white matter hyperintensity (WMH) $[6,7]$ and brain infarcts (BI) [8-12], are very tightly related to risk of clinical stroke. However, there has been relatively little work on association of diet with these MRI markers. In this review, published observational studies and clinical trials that evaluated the association between dietary factors and MRI-assessed cerebrovascular disease (WMH and BI) are summarized (Table 1) and future directions of research are suggested. 

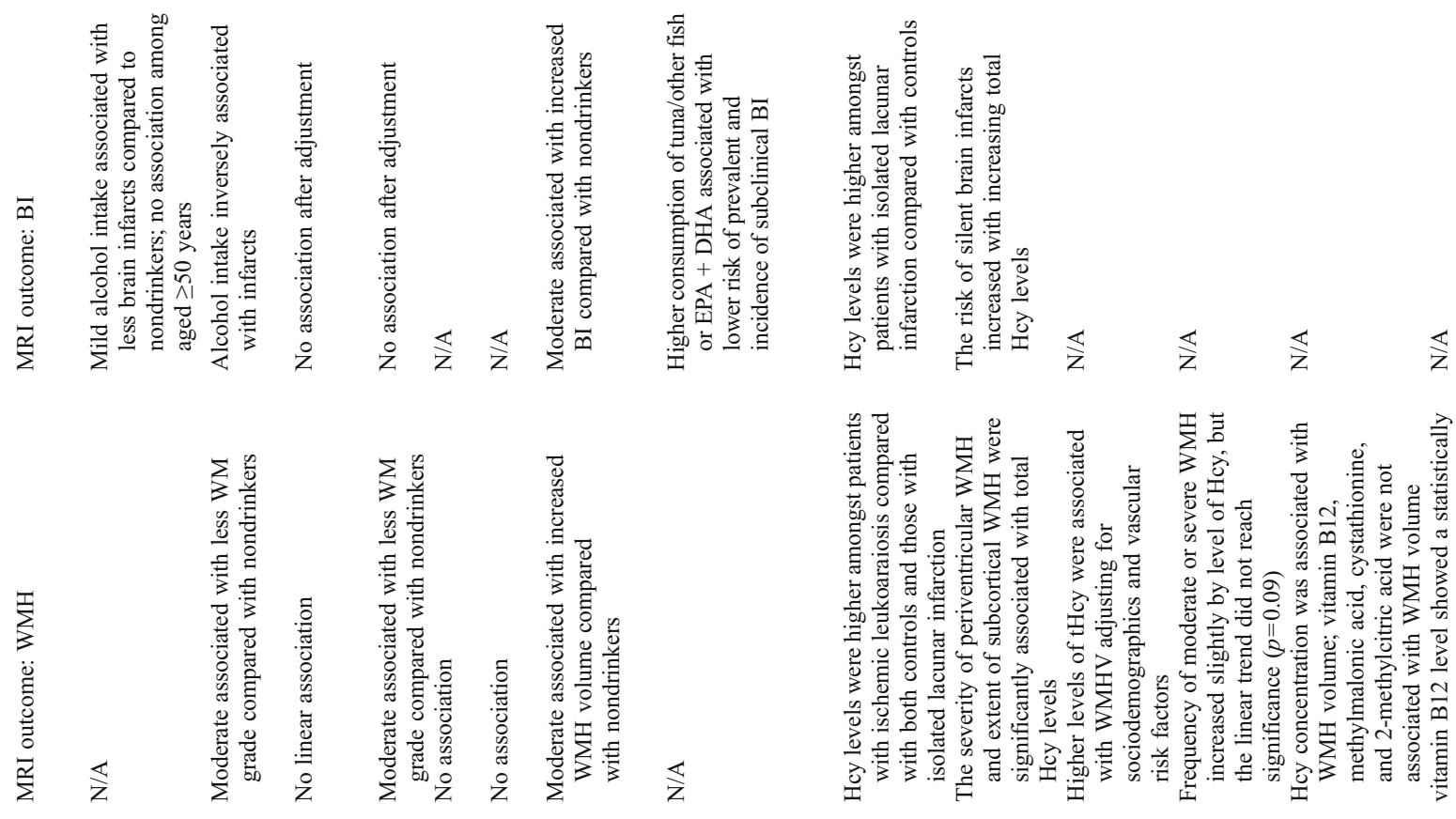

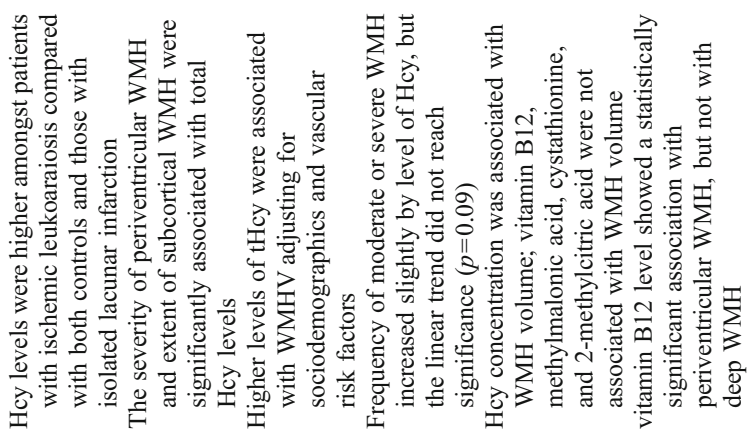
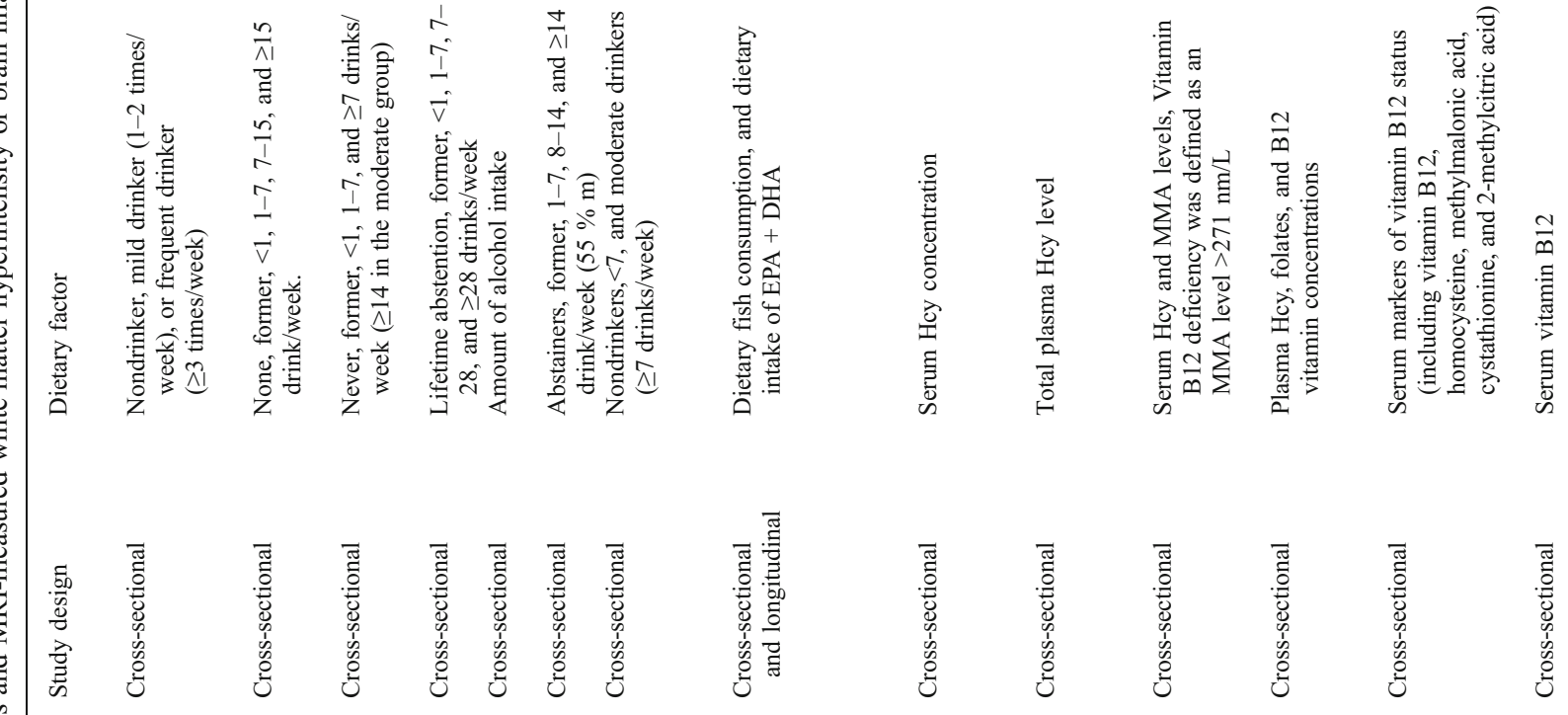

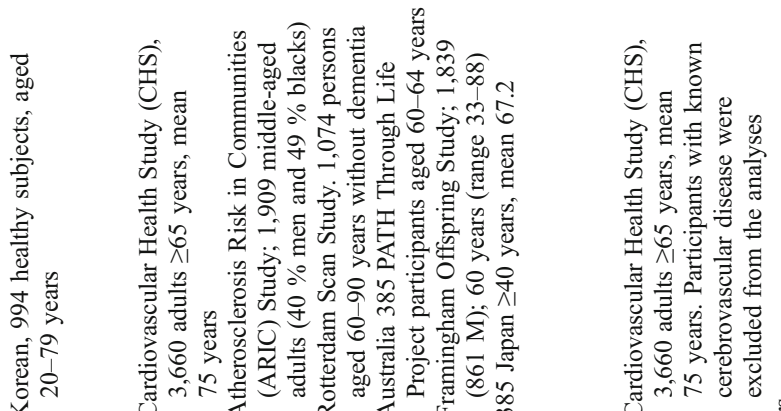

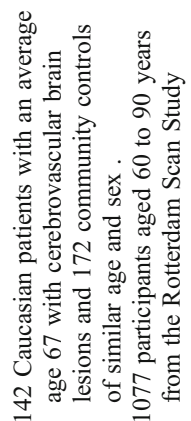

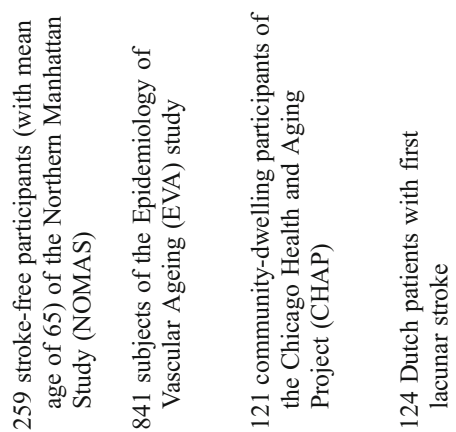

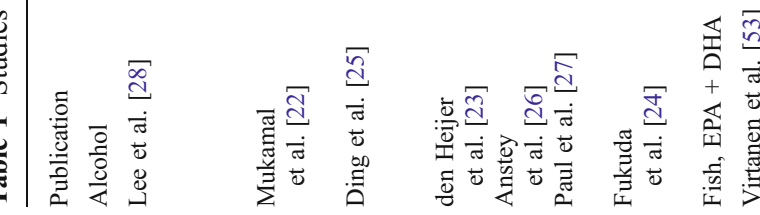

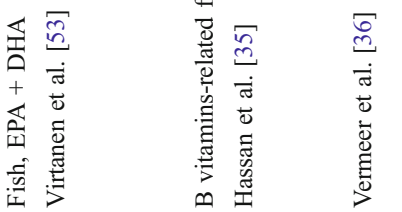

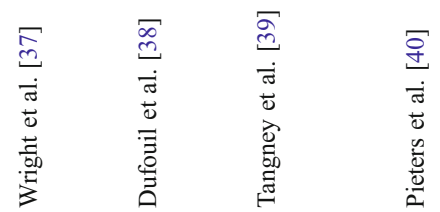




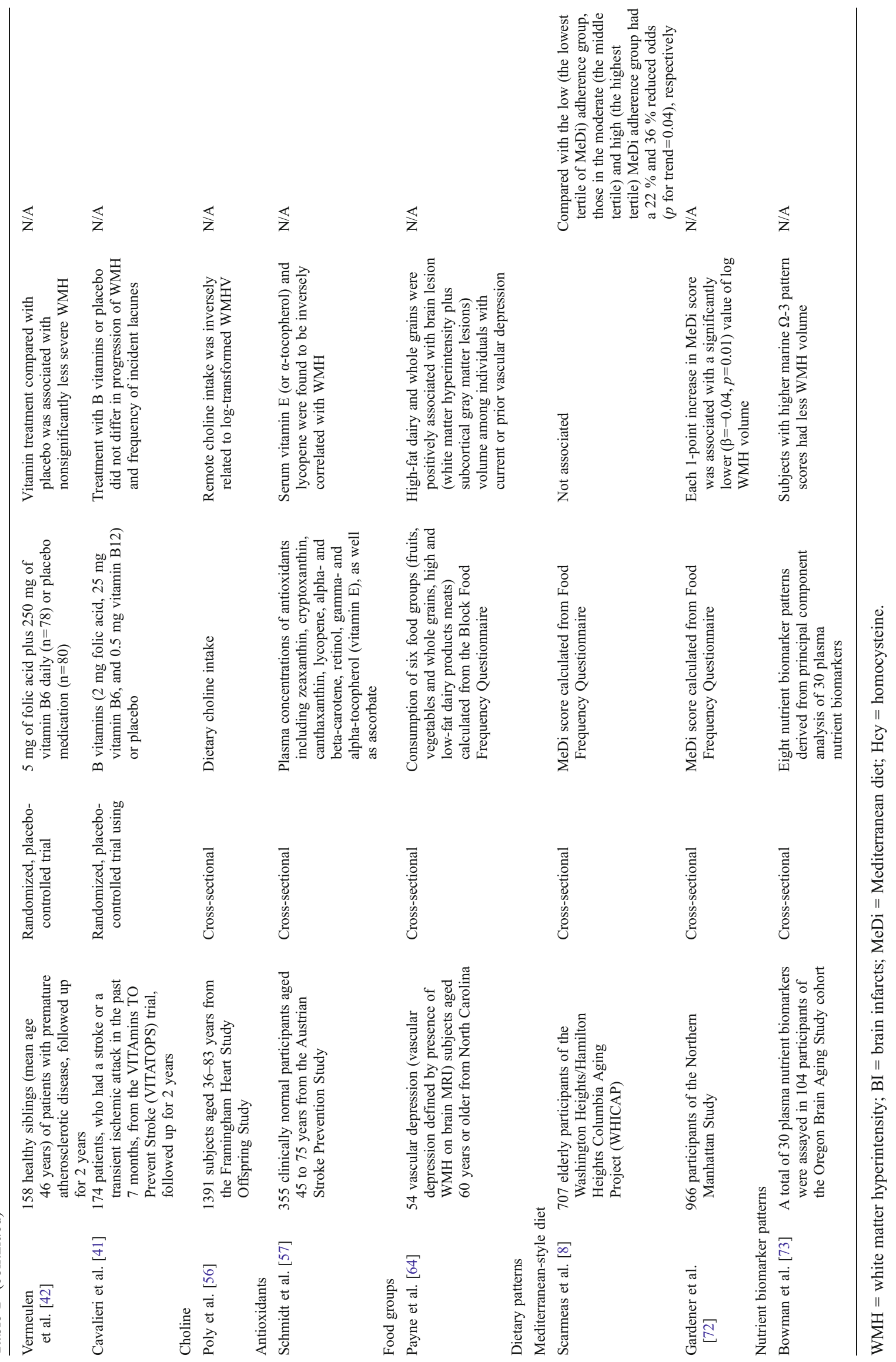




\section{MRI-Assessed Cerebrovascular Diseases}

BIs appear as punctate or circumscribed hypointense lesions on T1-weighted scans (and hyperintense in T2 and FLAIR sequences). Evidence from observational investigations suggests that increasing age, hypertension, and other cardiovascular risk factors are strongly associated with BI. Most BI are lacunar infarcts and small-vessel disease seems to play a crucial role in their pathogenesis [13]. Nevertheless, BIs larger than lacunes also may be noted.

$\mathrm{WMH}$, or leukoaraiosis, consist of bilateral patchy or diffuse areas of hyperintensity of the cerebral white matter on T2-weighted MRI images, including FLAIR sequences. Several reports suggest that the severity of WMH increases with age [14]. Although their pathogenesis is uncertain, $\mathrm{WMH}$ is traditionally thought to be a surrogate marker of small vessel vascular disease [15], resulting from ischemic damage due to chronic systemic hypoperfusion [16].

$\mathrm{BI}$ and WMH are highly related to each other [14], and they seem to represent two different manifestations of cerebral small-vessel disease [16] (although BI can be a manifestation of large vessel disease too). Overall, they are both associated with traditional vascular risk factors $[6,17,18]$ and often are associated with higher risk of subsequent clinical stroke $[19,20]$. Thus, modifying risk factors that contribute to the development of these brain changes seen in MRI may help the ultimate goal of preventing stroke.

\section{Epidemiological Evidence on Diet and Cerebrovascular Diseases}

\section{Alcohol}

Moderate drinking (1-2 drinks, or 10-30 g alcohol, per day) may be protective, whereas heavy drinking may be harmful for clinically diagnosed ischemic stroke (compared with nondrinking or drinking very small quantities) [21]. To date, several studies have examined the association between alcohol consumption and brain MRI markers among nonalcohol-dependent populations [22-27]. Three studies suggested a beneficial role of alcohol consumption on cerebrovascular diseases. In a cross-sectional study of 994 Korean symptom-free adults, history of mild alcohol consumption was associated with a low risk for silent cerebral infarction [28]. In 1992-1994, the association between alcohol use and subclinical brain findings was investigated among 3,660 participants of Cardiovascular Health Study (CHS) who completed cranial MRI examination. The study found that light to moderate alcohol drink in older adults was inversely associated with the presence MRI infarction and WMH compared with nondrinkers or heavy drinkers [22]. In the Rotterdam Scan
Study, an inverse U-shaped association between WMH, but not infarcts, and alcohol consumption was found among 1,074 nondemented older persons [23]. In contrast, a Japanese study of 385 adults found light and moderate alcohol drinkers had larger WMH volume and higher odds of having BI than nondrinking adults [24].

A few studies did not find any association between alcohol and MRI-assessed cerebrovascular diseases [25-27]. The associations of alcohol use with WMH and BI were examined in the Atherosclerosis Risk in Communities (ARIC) study, in which 1,920 adults aged 55-72 years underwent MRI. The study found that current drinking was not statistically associated with WMH or BI overall [25]. However, this study found that current drinking was associated with greater WMH among African Americans, but not among European Americans, suggesting a potential interaction between alcohol drinking and race [25]. Alcohol consumption was not associated with WMH among 385 elderly participants of the Australia PATH Through Life Project [26]. Similarly, no significant association between alcohol consumption and WMH was found among 1,839 subjects from the Framingham Offspring Study [27]. BI was not evaluated in the latter two studies [26, 27].

In summary, the evidence on the relationship between alcohol consumption on markers of cerebrovascular disease is inconsistent.

\section{B Vitamins}

Several large studies have found that intake of folate was associated with a significantly lower risk of clinical ischemic [29-31] or hemorrhagic stroke [32]. The Health Professionals Follow-up Study found that intake of vitamin B12 was marginally inversely associated with risk of ischemic stroke [29]. Furthermore, data from randomized trials suggested that folic acid supplementation with or without a combination with other B vitamins could reduce the risk of stroke [33]. Thus, it would be interesting to investigate whether B vitamins also are associated with MRI-assessed WMH or BI.

$\mathrm{B}$ vitamins (folic acid, vitamin B12, and B6) have been mainly related to cerebrovascular disease by their effect on lowering serum homocysteine (Hcy) levels, which is a strong risk factor for cardiovascular disease [34]. Several cross-sectional studies reported a direct association between elevated blood concentrations of total Hcy and greater burden of WMH volume or more BI [35-38]. In a recent longitudinal study of 121 community-dwelling participants of the Chicago Health and Aging Project (CHAP), the associations between serum markers of vitamin B12 status (including vitamin B12, Hcy, methylmalonic acid, cystathionine, and 2-methylcitric acid) and brain MRI measures were examined [39]. The study 
found that Hcy concentration was the only vitamin B12 indicator that was associated with WMH volume [39], but none of the vitamin B12 indicators were associated with cerebral infarcts [39]. In another study of 124 patients who had their first lacunar stroke, lower vitamin B12 level was significantly associated with more severe periventricular WMH but not with deep WMH [40].

However, data on the association between dietary intake or intake of supplements of B vitamins and $\mathrm{WMH}$ volume or BI were rare. Only two clinical trials examined the effect of supplements of B vitamins on MRI measured cerebrovascular lesions. In a substudy of the VITAmins TO Prevent Stroke (VITATOPS) trial, a prospective, randomized, double-blind, placebo-controlled, clinical trial, researchers assessed whether the once-daily supplements of B vitamins would reduce the progression of cerebrovascular brain lesions on MRI [41]. A total of 174 patients, who had a stroke (ischemic or hemorrhagic) or a transient ischemic attack in the past 7 months, were randomly assigned to receive either B vitamins or placebo. After 2 years of treatment, progression of $\mathrm{WMH}$ and frequency of incident lacunes were not significantly different between the two groups. However, in a post hoc subset analysis of 100 patients with MRI evidence of severe cerebrovascular lesions, B-vitamin supplementation was associated with a significant reduction in WMH volume change [41]. In another clinical trial using supplements of B vitamins in 158 healthy siblings of patients with premature atherosclerotic disease, B vitamins treatment, as compared to placebo, was nonsignificantly associated less white matter damage [42]. To date, no observational study has investigated whether $B$ vitamin intake is associated with lower risk of developing cerebrovascular lesions among general populations.

In summary, several observational studies on serum markers of vitamin B12 have suggested a potential beneficial role of B vitamins in MRI-assessed cerebrovascular disease among healthy elderly populations. The two clinical trials that showed some preliminary benefits of B vitamins supplementation in reducing the cerebrovascular lesion burden were among populations who had higher risk of stroke or among stroke patients. Nevertheless, whether such effect of supplementation of B vitamins also can be seen among healthy populations or whether dietary intake of B vitamins have similar effect on MRI-detected cerebrovascular disease has yet to be determined.

\section{Fish and Fatty Acids}

Many previous studies on fish consumption in relation to clinical stroke in general indicated that fish or unsaturated fatty acids consumption was inversely associated with risk of stroke [43-45], but negative studies also exist [46-52]. Whether fish consumption affects the risk of $\mathrm{WMH}, \mathrm{BI}$, or other MRI-measured cerebrovascular diseases is largely unknown and has only been explored in one populationbased study.

Researchers of the CHS investigated the cross-sectional associations of fish and dietary eicosapentaenoic acid (EPA) plus docosahexaenoic acid (DHA) consumption with MRIdefined subclinical brain abnormalities including infarcts WMH. They also explored longitudinal associations between fish and EPA + DHA consumption and subclinical BI. In the cross-sectional analysis that included more than 4,000 subjects, higher consuming tuna/other fish was associated with reduced risk of having subclinical BI or better white matter grade scores; in contrast, fried fish consumption was associated with nonsignificant trends toward higher prevalence of subclinical infarct. In the longitudinal analyses of a subgroup of 1,124 subjects, tuna/other fish consumption again tended to be associated with lower incidence of subclinical BI, whereas fried fish was not associated with risk of incident subclinical BI. Findings for estimated consumption of dietary EPA and DHA in this study were generally similar to those for consumption of tuna/other fish [53].

\section{Choline}

Choline is an essential nutrient that is a precursor of acetylcholine. It also serves as the precursor of sphingomyelin and phosphatidylcholine-structural components of cell membranes [54]. Main food sources of choline are red meat, poultry, milk, eggs, fish, and some beverages (coffee or beer) and fruit (orange) or vegetables (potatoes, broccoli) [55]. A recent study examined whether choline intake from foods was related with WMH level in a dementia-free cohort of 1,391 subjects from the Framingham Heart Study Offspring Study. The study found that although concurrent dietary intake of choline was not associated with $\mathrm{WMH}$ volume, choline intake in the past (approximately 7 years before the MRI measurement) was inversely related to logtransformed WMH volume or presence of large WMH [56].

\section{Antioxidants}

Only one study has examined the relationship between antioxidants blood level and cerebrovascular lesion. In the Austrian Stroke Prevention Study plasma concentrations of ten natural antioxidants, including zeaxanthin, cryptoxanthin, canthaxanthin, lycopene, alpha- and beta-carotene, retinol, gamma- and alpha-tocopherol (vitamin E), as well as ascorbate, were measured for 355 clinically healthy elderly participants [57]. The study found lower plasma alpha-tocopherol and lycopene were found to be correlated with a higher extent of MRI white matter abnormalities [57]. These findings were in line with the evidence that showed high serum alpha- 
tocopherol was associated with decreased the risk of clinical cerebral infarction [58]. Nevertheless, to our knowledge, no study has investigated directly the dietary intake of antioxidants and risk of cerebrovascular lesions using MRI. This may not be surprising, however, as previous investigations from large observational studies have yielded conflicting results with regard to whether dietary intakes of antioxidants may reduce the risk of clinical stroke [59-62]. Furthermore, clinical trials generally found that supplementation of these antioxidants did not prevent stroke [63].

\section{Food Groups}

The relationship between food groups and brain lesions has been examined in a study including 54 elderly vascular depression subjects (vascular depression defined by presence of WMH on brain MRI). Consumption of six food groups (fruits, vegetables and whole grains, high and low-fat dairy products meats) was calculated from the Block Food Frequency Questionnaire, and brain lesion (periventricular and deep $\mathrm{WMH}$, and subcortical gray matter) volumes were calculated from MRI. This study found that intakes of high-fat dairy and whole grains were positively associated with brain lesion volume among individuals with current or prior vascular depression, whereas there were no significant associations for vegetables, fruits, meats, and low-fat dairy products [64]. Saturated fat, a prominent component of fatty dairy foods, is known to be a cardiovascular risk factor. Thus, the findings regarding high-fat dairy products are consistent with the literature, given the ischemic nature of brain lesions seen in latelife depression. However, it is unknown why the whole grains were associated with higher burden of brain hyperintensive lesions; one would expect a protective effect of whole grains for ischemic brain lesions given the beneficial effects of whole grains on cardiovascular events [65]. It should be noted although that the study was small in terms of sample size, the study subjects were vascular depression patients rather than general population, and brain lesion included both grey and white matter hyperintensity. Further larger studies are needed.

\section{Dietary Patterns}

In addition to individual nutrients or food items, dietary patterns are continuously receiving attention, probably due to the ability of dietary patterns to represent complicated interactions and cumulative effects of nutrients or food items in a diet $[66,67]$.

\section{Mediterranean-Style Diet}

One of the dietary patterns that has been extensively studied is the Mediterranean-style diet (MeDi). The MeDi, representing the typical dietary habits of the populations bordering the Mediterranean Sea, is a diet high in plant foods (such as fruits, nuts, legumes, and cereals) and fish, with olive oil as the primary source of monounsaturated fat (MUFA) and low to moderate intake of wine, as well as low intake of red meat and poultry [68]. The MeDi has been associated with a number of healthful outcomes, including reduced risk of cardiovascular disease, cancer, and mortality $[69,70]$. In a recent, randomized trial of MeDi for the primary prevention of cardiovascular events in Spain, 7,447 participants who were at high cardiovascular risk, but with no cardiovascular disease at enrollment, were randomly assigned to one of three diets: a MeDi supplemented with extra-virgin olive oil, a MeDi supplemented with mixed nuts, or a low-fat diet. After a median follow-up of 4.8 years, a MeDi supplemented with extra-virgin olive oil or nuts reduced the incidence of stroke and other major cardiovascular events [71].

The relationship between $\mathrm{MeDi}$ and WMHV or BI has been explored in two community-based cohorts, both with participants residing in uptown New York. In the first study, BI and WMH were assessed using high-resolution structural MRI on 707 elderly participants of the Washington Heights/Hamilton Heights Columbia Aging Project (WHICAP) imaging substudy. The dietary assessments were administered an average of 5.8 years before the MRI. The study found that compared with the low (the lowest tertile of $\mathrm{MeDi}$ ), those in the moderate (the middle tertile) and high (the highest tertile) MeDi adherence groups had significantly reduced odds of MRI-assessed infarcts. No significant association between MeDi and WMH was found in this subgroup of WHICAP population [8]. Nevertheless, higher adherence to MeDi was associated with a lower WMH burden in another population, the Northern Manhattan Study. In this study of 966 participants, each 1-point increase in $\mathrm{MeDi}$ score was associated with a significantly lower $(\beta=-0.04, p=0.01)$ value of $\log \mathrm{WMH}$ volume [72]. In summary, both of these two studies suggested a possible protective association between increased adherence to a MeDi and MRI-assessed cerebrovascular diseases.

\section{Nutrient Biomarkers}

In a recent study, nutrient biomarker patterns were constructed to represent the combined effect of nutrients [73]. In this study, a total of 30 plasma nutrient biomarkers were assayed in 104 participants of the Oregon Brain Aging Study cohort. Principal component analysis was used to construct nutrient biomarker patterns. The study found that subjects with higher marine $\Omega-3$ pattern scores had less WMH volume, although the association was attenuated and no longer significant after adjustment for depression and hypertension [73]. These 
findings are consistent with the CHS study findings of a beneficial role of fish and EPA + DHA on CSVD [53].

\section{Conclusions}

Research on the associations between diet and neuroimaging markers of cerebrovascular lesions has been relatively sparse. The few existing studies examined only a handful individual nutrients or foods (dietary intake of alcohol [22-27], B vitamins [41, 42], fish [53], choline [56], serum markers of antioxidants [57]) and a few food groups [65], dietary patterns $[8,72]$, and nutrient biomarker patterns [74]. For most of these dietary factors, findings can be considered as inconclusive either due to conflicting results from different studies or due to lack of replication. Alcohol is the food item studied relatively commonly but results are quite mixed with protective [22, 23], detrimental [24], or no effect [25-27] of alcohol intake on cerebrovascular lesions all being reported. Available evidence for B vitamins, fish, choline, and antioxidants usually come from one or two studies. Results from dietary pattern analysis tended to suggest a potential protective role of $\mathrm{MeDi}$ or a dietary pattern high in marine $\Omega-3$ consumption, but more studies are needed to confirm these findings. Therefore, for most of the dietary factors that have been examined, further study is necessary to replicate the existing findings and to determine the role that these foods may play on the preservation of brain health.

Future studies may need to consider many other foods or nutrients in light of their association with clinical stroke. For example, long-term coffee drinking [75, 76], tea consumption [77], and dairy consumption [78-83] have all been associated with reduced risk of stroke, but no studies have examined how consumption of these foods may impact the MRI-measured BI or WMH. Besides MeDi, there are many ways to extract dietary patterns that could best represent the eating habits in a population. Available dietary pattern analysis methods, such as reduced rank regression analysis [84] and cluster analysis [85], have yet to be applied in this area.

In terms of study design, most of the past observational studies had a cross-sectional design, which limits assumptions of an etiological effect of diet on brain lesions. The effect of diet on longitudinal changes in imaging markers over time has been rarely studied. Some intervention studies are exceptions to this but they are quite few.

In summary, current evidence regarding the role of dietary factors on MRI-assessed cerebrovascular lesions is limited. Future studies are needed to confirm the previous findings, explore new dietary factors, and apply longitudinal study design.
Acknowledgments Nikolaos Scarmeas is supported by grants PO1-AG07232, AG028506, and Alzheimer's Association grant IIRG-09-133014.

Sources of Sponsorship PO1-AG07232, AG028506, Alzheimer's Association grant IIRG-09-133014.

Conflict of Interest Yian Gu declares that she has no conflict of interest. Nikolaos Scarmeas declares that he has no conflict of interest.

\section{References}

Papers of particular interest, published recently, have been highlighted as:

•• Of major importance

1. Feigin VL et al. Stroke epidemiology: a review of populationbased studies of incidence, prevalence, and case-fatality in the late 20th century. Lancet Neurol. 2003;2(1):43-53.

2. Kokubo Y. Traditional risk factor management for stroke: a neverending challenge for health behaviors of diet and physical activity. Curr Opin Neurol. 2012;25(1):11-7.

3. - Hampel $\mathrm{H}$ et al. Core candidate neurochemical and imaging biomarkers of Alzheimer's disease. Alzheimer's Dement. 2008;4(1):38-48. This paper was presented in part by the 1st author at the 10th International Conference of Alzheimer's Disease and Related Disorders (ICAD), Madrid, Spain, July 2006, as an invited plenary lecture.

4. Longstreth Jr WT et al. Incidence, manifestations, and predictors of brain infarcts defined by serial cranial magnetic resonance imaging in the elderly: the cardiovascular health study. Stroke. 2002;33(10):2376-82.

5. Reitz $\mathrm{C}$ et al. Validity of self-reported stroke in elderly African Americans, Caribbean Hispanics, and whites. Arch Neurol. 2009;66(7):834-40.

6. Longstreth Jr WT et al. Clinical correlates of white matter findings on cranial magnetic resonance imaging of 3301 elderly people: the cardiovascular health study. Stroke. 1996; 27(8):1274-82.

7. Brickman AM et al. Measuring cerebral atrophy and white matter hyperintensity burden to predict the rate of cognitive decline in Alzheimer disease. Arch Neurol. 2008;65(9):1202-8.

8. Scarmeas $\mathrm{N}$ et al. Mediterranean diet and magnetic resonance imagingassessed cerebrovascular disease. Ann Neurol. 2011;69(2):257-68.

9. Price TR et al. Silent brain infarction on magnetic resonance imaging and neurological abnormalities in community-dwelling older adults. The cardiovascular health study. CHS collaborative research group. Stroke. 1997;28(6):1158-64.

10. Lopez OL et al. Risk factors for mild cognitive impairment in the cardiovascular health study cognition study. Arch Neurol. 2003;60(10):1394-9.

11. Vermeer SE et al. Silent brain infarcts and the risk of dementia and cognitive decline. N Engl J Med. 2003;348(13):1215-22.

12. Luchsinger JA et al. Subclinical cerebrovascular disease in mild cognitive impairment. Neurology. 2009;73(6):450-6.

13. Vermeer SE, Longstreth Jr WT, Koudstaal PJ. Silent brain infarcts: a systematic review. Lancet Neurol. 2007;6(7):611-9.

14. DeCarli $\mathrm{C}$ et al. Measures of brain morphology and infarction in the framingham heart study: establishing what is normal. Neurobiol Aging. 2005;26(4):491-510. 
15. Pantoni L, Poggesi A, Inzitari D. The relation between white-matter lesions and cognition. Curr Opin Neurol. 2007;20(4):390-7.

16. Pantoni L, Garcia JH. Pathogenesis of leukoaraiosis: a review. Stroke. 1997;28(3):652-9.

17. DeCarli $\mathrm{C}$ et al. Cerebrovascular and brain morphologic correlates of mild cognitive impairment in the national heart, lung, and blood institute twin study. Arch Neurol. 2001;58(4):643-7.

18. Inzitari D et al. Vascular risk factors and leuko-araiosis. Arch Neurol. 1987;44(1):42-7.

19. Vermeer SE et al. Silent brain infarcts and white matter lesions increase stroke risk in the general population: the Rotterdam scan study. Stroke. 2003;34(5):1126-9.

20. Debette $\mathrm{S}$ et al. Association of MRI markers of vascular brain injury with incident stroke, mild cognitive impairment, dementia, and mortality: the Framingham offspring study. Stroke. 2010;41(4):600-6.

21. Patra $J$ et al. Alcohol consumption and the risk of morbidity and mortality for different stroke types-a systematic review and metaanalysis. BMC Publ Health. 2010;10:258.

22. Mukamal KJ et al. Alcohol consumption and subclinical findings on magnetic resonance imaging of the brain in older adults: the cardiovascular health study. Stroke. 2001;32(9):1939-46.

23. den Heijer $\mathrm{T}$ et al. Alcohol intake in relation to brain magnetic resonance imaging findings in older persons without dementia. Am J Clin Nutr. 2004;80(4):992-7.

24. Fukuda $\mathrm{K}$ et al. Alcohol intake and quantitative MRI findings among community dwelling Japanese subjects. J Neurol Sci. 2009;278(1-2):30-4.

25 . Ding J et al. Alcohol intake and cerebral abnormalities on magnetic resonance imaging in a community-based population of middleaged adults: the Atherosclerosis Risk in Communities (ARIC) study. Stroke. 2004;35(1):16-21.

26. Anstey KJ et al. Weekly alcohol consumption, brain atrophy, and white matter hyperintensities in a community-based sample aged 60 to 64 years. Psychosom Med. 2006;68(5):778-85.

27. Paul CA et al. Association of alcohol consumption with brain volume in the Framingham study. Arch Neurol. 2008;65(10):1363-7.

28. Lee SC et al. Prevalence and risk factors of silent cerebral infarction in apparently normal adults. Hypertension. 2000;36(1):73-7.

29. He K et al. Folate, vitamin B6, and B12 intakes in relation to risk of stroke among men. Stroke. 2004;35(1):169-74.

30. Larsson SC et al. Folate, vitamin B6, vitamin B12, and methionine intakes and risk of stroke subtypes in male smokers. Am J Epidemiol. 2008;167(8):954-61.

31. Weng LC et al. Is ischemic stroke risk related to folate status or other nutrients correlated with folate intake? Stroke. 2008;39(12):3152-8.

32. Van Guelpen B et al. Folate, vitamin B12, and risk of ischemic and hemorrhagic stroke: a prospective, nested case-referent study of plasma concentrations and dietary intake. Stroke. 2005;36(7):1426-31.

33. Huang $\mathrm{T}$ et al. Meta-analysis of $\mathrm{B}$ vitamin supplementation on plasma homocysteine, cardiovascular and all-cause mortality. Clin Nutr. 2012;31(4):448-54.

34. Refsum $\mathrm{H}$ et al. Homocysteine and cardiovascular disease. Annu Rev Med. 1998;49:31-62.

35. Hassan A et al. Homocysteine is a risk factor for cerebral small vessel disease, acting via endothelial dysfunction. Brain. 2004; 127(Pt 1):212-9.

36. Vermeer $\mathrm{SE}$ et al. Homocysteine, silent brain infarcts, and white matter lesions: the Rotterdam scan study. Ann Neurol. 2002;51(3):285-9.

37. Wright $\mathrm{CB}$ et al. Total homocysteine is associated with white matter hyperintensity volume: the Northern Manhattan study. Stroke. 2005;36(6):1207-11.

38. Dufouil $\mathrm{C}$ et al. Homocysteine, white matter hyperintensities, and cognition in healthy elderly people. Ann Neurol. 2003;53(2):214-21.

39. Tangney CC et al. Vitamin B12, cognition, and brain MRI measures: a cross-sectional examination. Neurology. 2011;77(13):1276-82.
40. Pieters B et al. Periventricular white matter lucencies relate to low vitamin B12 levels in patients with small vessel stroke. Stroke. 2009;40(5):1623-6.

41. Cavalieri $\mathrm{M}$ et al. $\mathrm{B}$ vitamins and magnetic resonance imagingdetected ischemic brain lesions in patients with recent transient ischemic attack or stroke: the VITAmins TO Prevent Stroke (VITATOPS) MRI-substudy. Stroke. 2012;43(12):3266-70.

42. Vermeulen EG et al. Effect of homocysteine-lowering treatment with folic acid plus vitamin B on cerebrovascular atherosclerosis and white matter abnormalities as determined by MRA and MRI: a placebo-controlled, randomized trial. Eur J Clin Invest. 2004;34(4):256-61.

43. He K. Dietary fat and stroke: a different story from coronary heart disease. Ital Heart J. 2003;4(12):821-3.

44. Mozaffarian D et al. Fish consumption and stroke risk in elderly individuals: the cardiovascular health study. Arch Intern Med. 2005; 165(2):200-6.

45. Laaksonen DE et al. Prediction of cardiovascular mortality in middle-aged men by dietary and serum linoleic and polyunsaturated fatty acids. Arch Intern Med. 2005;165(2):193-9.

46. Orencia AJ et al. Fish consumption and stroke in men. 30-year findings of the Chicago western electric study. Stroke. 1996;27(2):204-9.

47. Keli SO, Feskens EJ, Kromhout D. Fish consumption and risk of stroke. The Zutphen study. Stroke. 1994;25(2):328-32.

48. Yuan JM et al. Fish and shellfish consumption in relation to death from myocardial infarction among men in Shanghai, China. Am J Epidemiol. 2001;154(9):809-16.

49. Folsom AR, Demissie Z. Fish intake, marine omega-3 fatty acids, and mortality in a cohort of postmenopausal women. Am J Epidemiol. 2004;160(10):1005-10.

50. Morris $\mathrm{MC}$ et al. Fish consumption and cardiovascular disease in the physicians' health study: a prospective study. Am J Epidemiol. 1995;142(2):166-75.

51. Montonen $\mathrm{J}$ et al. Fish consumption and the incidence of cerebrovascular disease. Br J Nutr. 2009;102(5):750-6.

52. Bravata DM et al. Dietary fish or seafood consumption is not related to cerebrovascular disease risk in twin veterans. Neuroepidemiology. 2007;28(3):186-90.

53. Virtanen JK et al. Fish consumption and risk of subclinical brain abnormalities on MRI in older adults. Neurology. 2008;71(6):439-46.

54. Zeisel SH, da Costa KA. Choline: an essential nutrient for public health. Nutr Rev. 2009;67(11):615-23.

55. Cho E et al. Dietary choline and betaine assessed by foodfrequency questionnaire in relation to plasma total homocysteine concentration in the Framingham offspring study. Am J Clin Nutr. 2006;83(4):905-11.

56. Poly $\mathrm{C}$ et al. The relation of dietary choline to cognitive performance and white-matter hyperintensity in the Framingham offspring cohort. Am J Clin Nutr. 2011;94(6):1584-91.

57. Schmidt R, Hayn M, Fazekas F et al. Magnetic resonance imaging white matter hyperintensities in clinically normal elderly individuals. Correlations with plasma concentrations of naturally occurring antioxidants. Stroke. 1996;27(11):2043-7.

58. Leppala JM et al. Different risk factors for different stroke subtypes: association of blood pressure, cholesterol, and antioxidants. Stroke. 1999;30(12):2535-40.

59. Ascherio A et al. Relation of consumption of vitamin E, vitamin C, and carotenoids to risk for stroke among men in the United States. Ann Intern Med. 1999;130(12):963-70.

60. Hirvonen $\mathrm{T}$ et al. Intake of flavonoids, carotenoids, vitamins $\mathrm{C}$ and $\mathrm{E}$, and risk of stroke in male smokers. Stroke. 2000; 31(10):2301-6.

61. Yochum L et al. Dietary flavonoid intake and risk of cardiovascular disease in postmenopausal women. Am J Epidemiol. 1999;149(10):943-9.

62. Voko $\mathrm{Z}$ et al. Dietary antioxidants and the risk of ischemic stroke: the Rotterdam study. Neurology. 2003;61(9):1273-5. 
63. Hankey GJ. Nutrition and the risk of stroke. Lancet Neurol. 2012;11(1):66-81.

64. Payne ME et al. Food group intake and brain lesions in latelife vascular depression. International Psychogeriatrics. 2007; 19(2):295-305

65. Jacobs DR Jr, Meyer KA, Kushi LH, Folsom AR. Whole-grain intake may reduce the risk of ischemic heart disease death in postmenopausal women: the Iowa Women's Health Study. Am J Clin Nutr. 1998;68(2):248-57.

66. Hu FB. Dietary pattern analysis: a new direction in nutritional epidemiology. Curr Opin Lipidol. 2002;13(1):3-9.

67. Gu Y, Scarmeas N. Dietary patterns in Alzheimer's disease and cognitive aging. Curr Alzheimer Res. 2011;8(5):510-9.

68. Roman B et al. Effectiveness of the Mediterranean diet in the elderly. Clin Interv Aging. 2008;3(1):97-109.

69. Babio N, Bullo M, Salas-Salvado J. Mediterranean diet and metabolic syndrome: the evidence. Public Health Nutr. 2009;12(9A):1607-17.

70. Sofi $\mathrm{F}$ et al. Adherence to Mediterranean diet and health status: meta-analysis. BMJ. 2008;337:a1344.

71. Estruch R, Ros E, Salas-Salvadó J et al. Primary prevention of cardiovascular disease with a Mediterranean diet. N Engl J Med. 2013;368(14):1279-90.

72. Gardener $\mathrm{H}$ et al. Mediterranean diet and white matter hyperintensity volume in the Northern Manhattan study. Arch Neurol. 2012;69(2):251-6.

73. Bowman GL et al. Nutrient biomarker patterns, cognitive function, and MRI measures of brain aging. Neurology. 2012;78(4):241-9.

74. Witte AV et al. Caloric restriction improves memory in elderly humans. Proc Natl Acad Sci U S A. 2009;106(4):1255-60.
75. Lopez-Garcia E et al. Coffee consumption and risk of stroke in women. Circulation. 2009;119(8):1116-23.

76. Larsson SC et al. Coffee and tea consumption and risk of stroke subtypes in male smokers. Stroke. 2008;39(6):1681-7.

77. Arab L, Liu W, Elashoff D. Green and black tea consumption and risk of stroke: a meta-analysis. Stroke. 2009;40(5):178692.

78. Warensjo E et al. Stroke and plasma markers of milk fat intake-a prospective nested case-control study. Nutr J. 2009;8:21.

79. Iso $\mathrm{H}$ et al. Prospective study of calcium, potassium, and magnesium intake and risk of stroke in women. Stroke. 1999; 30(9):1772-9.

80. Abbott RD et al. Effect of dietary calcium and milk consumption on risk of thromboembolic stroke in older middle-aged men. The Honolulu Heart Program Stroke. 1996;27(5):813-8.

81. Kinjo $Y$ et al. Possible protective effect of milk, meat and fish for cerebrovascular disease mortality in Japan. J Epidemiol. 1999;9(4):268-74.

82. Umesawa $M$ et al. Dietary intake of calcium in relation to mortality from cardiovascular disease: the JACC study. Stroke. 2006;37(1):20-6.

83. Umesawa $\mathrm{M}$ et al. Dietary calcium intake and risks of stroke, its subtypes, and coronary heart disease in Japanese: the JPHC study cohort I. Stroke. 2008;39(9):2449-56.

84. Gu Y et al. Food combination and Alzheimer's disease risk: a protective diet. Arch Neurol. 2010;67(6):699-706.

85. Samieri C et al. Dietary patterns derived by hybrid clustering method in older people: association with cognition, mood, and self-rated health. J Am Diet Assoc. 2008;108(9):1461-71. 